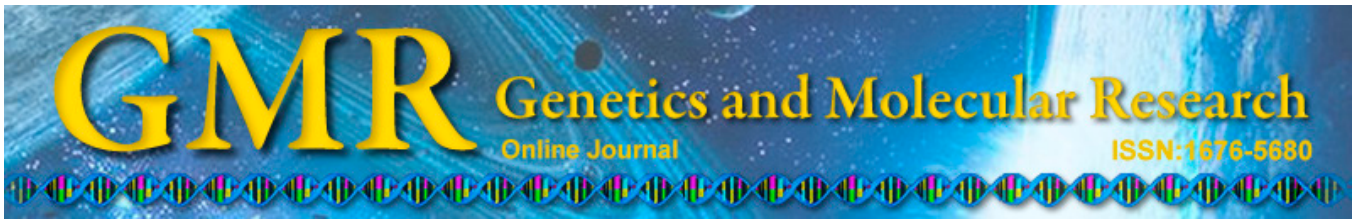

\title{
Effects of high intensity exhaustive exercise on SOD, MDA, and NO levels in rats with knee osteoarthritis
}

\author{
X.D. Li ${ }^{1}$, G.F. Sun ${ }^{1}$, W.B. Zhu' ${ }^{2}$ and Y.H. Wang ${ }^{1}$ \\ 'Department of Orthopedic Trauma, Binzhou Municipal People's Hospital, \\ Binzhou, Shandong, China \\ ${ }^{2}$ Department of Anesthesiology, Binzhou Municipal People's Hospital, \\ Binzhou, Shandong, China \\ Corresponding author: X.D. Li \\ E-mail: XiaodongLi1976@126.com
}

Genet. Mol. Res. 14 (4): 12367-12376 (2015)

Received January 15, 2015

Accepted June 3, 2015

Published October 16, 2015

DOI http://dx.doi.org/10.4238/2015.October.16.3

\begin{abstract}
The aim of this study was to investigate the impact of high intensity exhaustive exercise on nitric oxide (NO), malondialdehyde (MDA), and superoxide dismutase (SOD) expression in rats with knee osteoarthritis. Sprague Dawley rats were randomly divided into control $(\mathrm{N}=5)$ and model $(\mathrm{N}=35)$ groups; the model group was further divided into quiet $(\mathrm{N}=5)$, low- $(\mathrm{N}=15)$ and high- $(\mathrm{N}=15)$ intensity exhaustive exercise groups. The low- and high-intensity groups were randomly divided into pre-exercise $(\mathrm{N}=5)$, immediate post-exercise $(\mathrm{N}=5)$, and 24-h post-exercise $(\mathrm{N}=5)$ groups according to different time points for detection. NO, MDA, and SOD levels were compared between each group. The SOD levels in the quiet, low-, and high-intensity exhaustive exercise groups were lower than that in the control group, whereas the NO and MDA levels were higher in the former groups than in the controls $(\mathrm{P}<0.05)$. The SOD level in the 24 -h post-low intensity exhaustive exercise group was higher than that in the 24-h post-high intensity exhaustive exercise group, whereas the NO and MDA levels
\end{abstract}


were lower in the 24-h post-low intensity than in the post-high intensity exercise group $(\mathrm{P}<0.05)$. Overall, the results demonstrated that with the increase of exercise intensity, the SOD activity in the rats with knee osteoarthritis decreased gradually, whereas the MDA and NO levels gradually increased. Thus, the greater the exercise intensity, the more serious the impact on knee osteoarthritis.

Key words: Knee osteoarthritis; High intensity exhaustive exercise; Superoxide dismutase; Malondialdehyde; Nitric oxide

\section{INTRODUCTION}

Osteoarthritis, also known as degenerative arthritis or joint disease, is a kind of non-inflammatory chronic articular disease characterized by bone hyperplasia and articular cartilage destruction or degeneration. Osteoarthritis is a common and frequently-occurring disease. Patients with early onset usually exhibit no obvious symptoms, whereas some patients present certain symptoms such as low local temperature or stiff discomfort of the knee; such symptoms can improve after activity. As the disease progresses, pain and difficulties standing up and climbing stairs might gradually appear. Some patients with severe osteoarthritis develop joint deformities if not treated in a timely manner (Toklu et al., 2010). Generally, osteoarthritis is related to many factors including immunity, heredity, metabolic disorder, obesity, trauma, and increased age (Lu et al., 2010). With the accelerated development of research on osteoarthritis, it has been found that cytokines, free radicals, nitric oxide (NO), and malondialdehyde (MDA) levels demonstrated close relationships with articular cartilage degeneration. Furthermore, exercise rehabilitation in osteoarthritis has become a popular topic for clinical research. Thus, our study analyzed the impact of high intensity exhaustive exercise on NO, MDA, and superoxide dismutase (SOD) expression in the knee arthritis rat model, with the aim of providing information for improving knee osteoarthritis patient rehabilitation therapy.

\section{MATERIAL AND METHODS}

\section{General information and animal usage}

Rats were used for all experiments, and all procedures were approved by the Animal Ethics Committee of Binzhou Municipal People's Hospital (Shandong, China). Forty male Sprague Dawley rats weighing 180-220 g were provided by the Binzhou City Animal Center. The experiments began after rats received adaptable feed for one week. The rats were randomly divided into control $(\mathrm{N}=5)$ and model $(\mathrm{N}=35)$ groups, and the model group was further divided into quiet $(\mathrm{N}=5)$, low intensity $(\mathrm{N}=15)$, and high-intensity exhaustive exercise ( $\mathrm{N}$ $=15$ ) groups. The low- and high-intensity exhaustive exercise groups were randomly divided into three subgroups including a pre-exercise $(\mathrm{N}=5)$, immediate post-exercise $(\mathrm{N}=5)$, and 24-h post-exercise $(\mathrm{N}=5)$ group according to different time points for detection.

Reagents utilized included NO, MDA, and SOD detection kits purchased from Chenguang Biotech Group Co., Ltd. (Quzhou, China), papain, and chloral hydrate. 


\section{Methods}

\section{Rat knee osteoarthritis modeling}

As described by Panicker et al. (2009), after the rats were successfully anesthetized with chloral hydrate, $0.2 \mathrm{~mL} 4 \%$ papain solution was injected into the knee joint cavities every two days for two weeks. X-ray and ultrasound screening were used for monitoring after the administration of the medication. Observation of knee joint cartilage degeneration conforming to types of osteoarthritis change was judged as modeling success.

\section{Exhaustive exercise}

After successful establishment of the knee osteoarthritis model, low or high intensity exhaustive exercises were performed by the rats according to Bedford animal motion model standards (Bedford et al., 1979). An electric treadmill (Yuyan Instrument, Shanghai, China) with a $10^{\circ}$ slope was used. Rats in the low intensity exhaustive exercise group began to run at 5 $\mathrm{m} / \mathrm{min}$ velocity for $15 \mathrm{~min}$, and then movement speed was gradually increased to a maximum of $15 \mathrm{~m} / \mathrm{min}$ until exhaustion. Rats in the high intensive exhaustive exercise group began to run at $15 \mathrm{~m} / \mathrm{min}$ velocity for $15 \mathrm{~min}$, and then movement speed was gradually increased to a maximum of $35 \mathrm{~m} / \mathrm{min}$ until exhaustion.

\section{Sampling}

Rats in the control and quiet groups were anaesthetized under quiet conditions, and $5 \mathrm{~mL}$ abdominal venous blood was collected. Rats in the low- and high-intensity exhaustive exercise groups were sampled at the different time points previously described. The blood samples were centrifuged at $3000 \mathrm{r} / \mathrm{min}$ for $5 \mathrm{~min}$ and the supernatants were collected and stored at $-20^{\circ} \mathrm{C}$.

\section{Index detection}

NO levels were detected by the nitrate reductase method; MDA levels were detected by the thiobarbituric acid method; and SOD levels were detected by the xanthine oxidase method. All detection procedures were in strict accordance with kit instructions.

\section{Statistical analysis}

The SPSS 20.0 statistical software (SPSS, Chicago, IL, USA) was used for data analyses. Data counts were analyzed by the $\chi^{2}$ test; measurement data are reported as means $\pm \mathrm{SE}$ and compared by analysis of variance (ANOVA) among different groups. Bonferroni's correction was applied for pairwise comparison, with statistically significant differences defined as $\mathrm{P}<0.05$.

\section{RESULTS}

\section{SOD expression in the different groups}

SOD levels in the quiet, low intensity, and high intensity exhaustive exercise groups 
were all significantly lower than that in the control group $(\mathrm{P}<0.05)$. The SOD level in the low intensity exhaustive exercise group 24-h post-exercise was higher than that in high intensity exhaustive exercise group $(\mathrm{P}<0.05)$ (Table 1$)$. The SOD levels in the low- and high-intensity exhaustive exercise groups are shown in Figure 1.

\begin{tabular}{lcc} 
Table 1. SOD levels in each groups (means $\pm \mathrm{SE}, \mathrm{U} / \mathrm{mL}$ ). \\
\hline Group & Patients & SOD \\
\hline Control & 5 & $45.79 \pm 14.84$ \\
Quiet & 5 & $33.32 \pm 8.52^{1}$ \\
Low-intensity exhaustive exercise & 15 & $33.67 \pm 5.73^{1}$ \\
Pre-exercise & 5 & $20.36 \pm 5.41^{1,2}$ \\
Immediate post-exercise & 5 & $29.33 \pm 4.98^{1}$ \\
24 h post-exercise & 5 & $33.14 \pm 6.55^{1}$ \\
High-intensity exhaustive exercise & 15 & $18.53 \pm 3.29^{1,2}$ \\
Pre-exercise & 5 & $21.41 \pm 6.09^{1,2}$ \\
Immediate post-exercise & 5 & 5 \\
24 h post-exercise & 5 &
\end{tabular}

${ }^{1}$ Compared with controls, $\mathrm{P}<0.05 ;{ }^{2}$ compared with pre-exercise, $\mathrm{P}<0.05$. SOD $=$ superoxide dismutase.

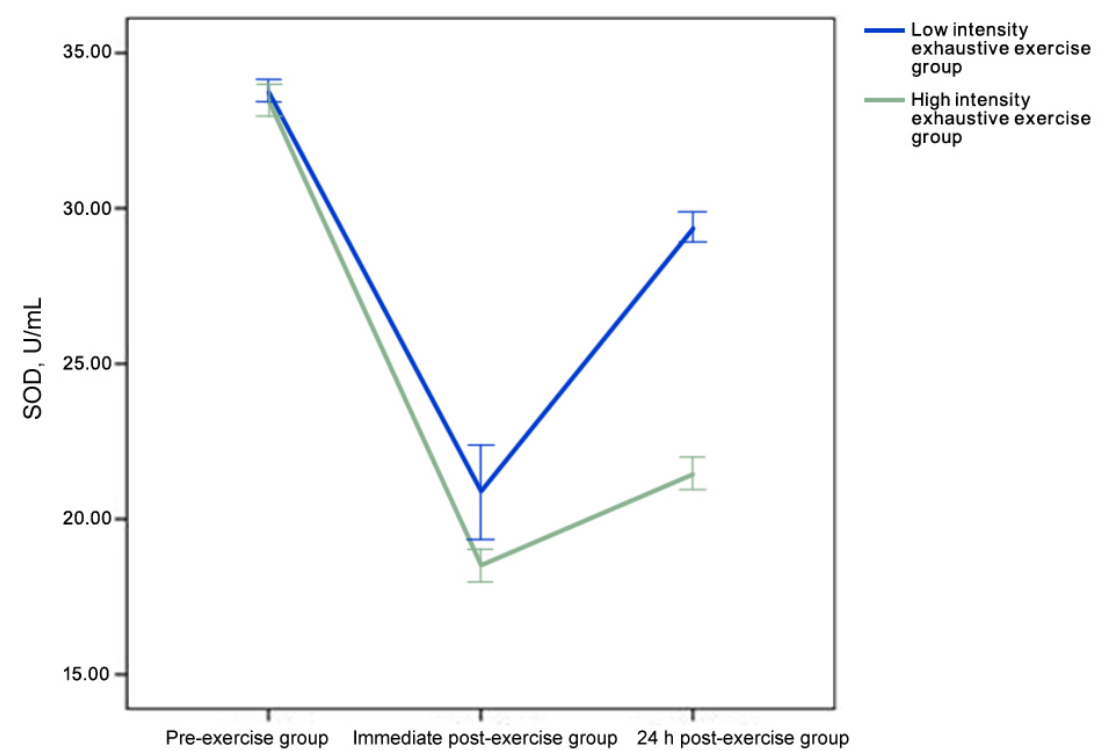

Figure 1. Superoxide dismutase (SOD) levels in the low- and high-intensity exhaustive exercise groups.

\section{MDA expression in the different groups}

MDA levels in the quiet, low intensity, and high intensity exhaustive exercise groups were all significantly higher than those in the control group $(\mathrm{P}<0.05)$. The MDA level in the low-intensity exhaustive exercise group $24 \mathrm{~h}$ post-exercise was lower than that in the high-intensity exhaustive exercise group $(\mathrm{P}<0.05)$ (Table 2$)$. The MDA levels in the low- and high-intensity exhaustive exercise groups are shown in Figure 2. 


\section{Table 2. MDA levels in each group (means $\pm \mathrm{SE}$, nom $/ \mathrm{mL}$ ).}

\begin{tabular}{lcc}
\hline Group & Patients & MDA \\
\hline Control & 5 & $5.95 \pm 1.51$ \\
Quiet & 5 & $10.48 \pm 2.89^{1}$ \\
Low-intensity exhaustive exercise & 15 & 5 \\
Pre-exercise & 5 & $10.04 \pm 2.77^{1}$ \\
Immediate post-exercise & 5 & $17.33 \pm 4.51^{1,2}$ \\
24 h post-exercise & 15 & $11.17 \pm 2.35^{2}$ \\
High-intensity exhaustive exercise & 5 & $10.21 \pm 3.04^{1}$ \\
Pre-exercise & 5 & $18.69 \pm 5.23^{1,2}$ \\
Immediate post-exercise & 5 & $16.64 \pm 3.06^{1,2}$ \\
24 h post-exercise & 5
\end{tabular}

${ }^{1}$ Compared with controls, $\mathrm{P}<0.05 ;{ }^{2}$ compared with pre-exercise, $\mathrm{P}<0.05 ; \mathrm{MDA}=$ malondialdehyde.

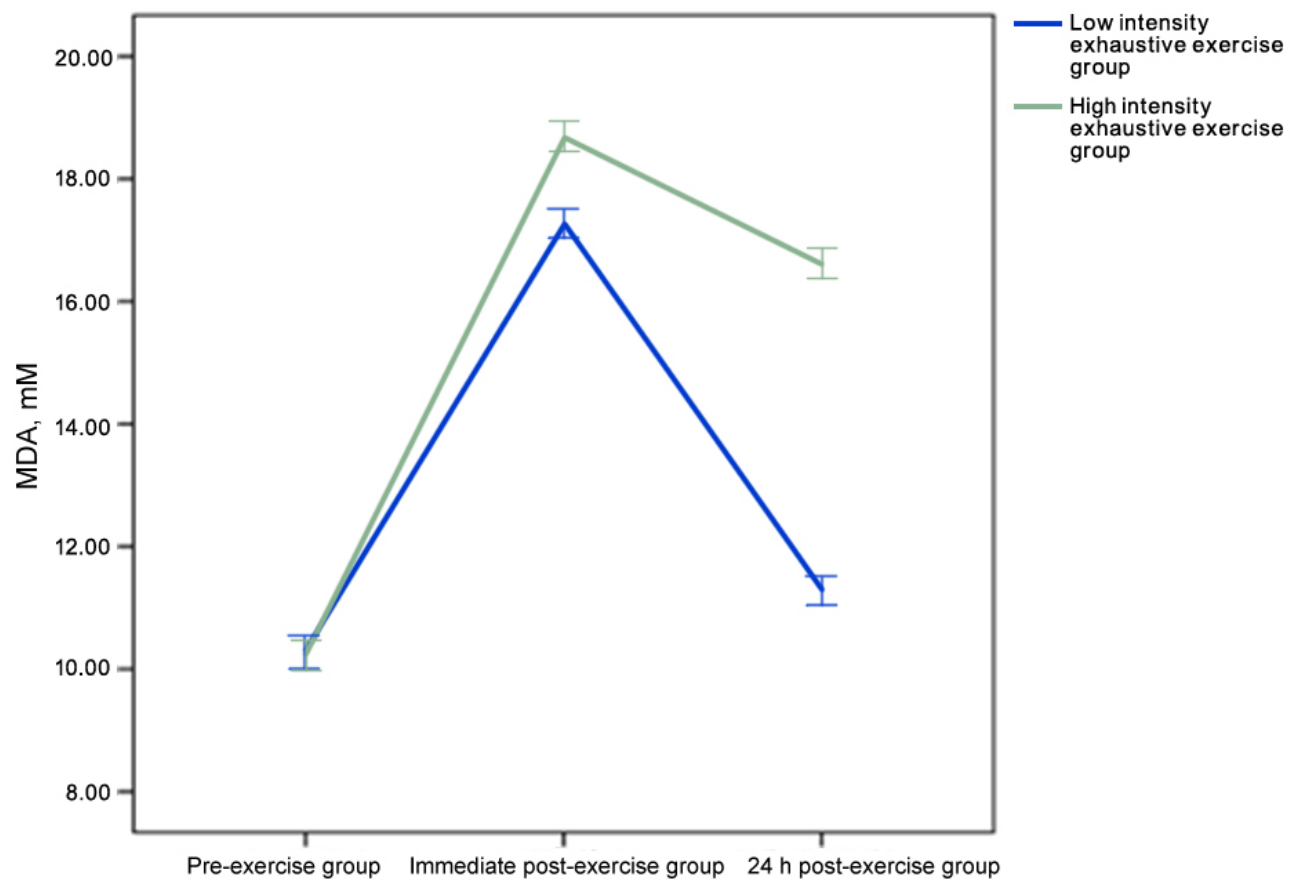

Figure 2. Malondialdehyde (MDA) levels in the low- and high-intensity exhaustive exercise groups.

\section{NO expression in the different groups}

NO levels in the quiet, low intensity, and high intensity exhaustive exercise groups were all significantly higher than that in the control group $(\mathrm{P}<0.05)$. The NO level in the lowintensity exhaustive exercise group $24 \mathrm{~h}$ post-exercise was lower than that in the high-intensity exhaustive exercise group $(\mathrm{P}<0.05)$ (Table 3$)$. The NO levels in the low- and high-intensity exhaustive exercise groups are shown in Figure 3. 


\begin{tabular}{lcc} 
Table 3. NO levels in each group (means $\pm \mathrm{SE}, \mu \mathrm{M})$. & & \\
\hline Group & Patients & NO \\
\hline Control & 5 & $40.58 \pm 4.19$ \\
Quiet & 15 & $67.32 \pm 6.24^{1}$ \\
Low-intensity exhaustive exercise & 5 & $66.59 \pm 6.51^{1}$ \\
Pre-exercise & 5 & $77.38 \pm 5.23^{1,2}$ \\
Immediate post-exercise & 5 & $68.47 \pm 6.85^{1}$ \\
24 h post-exercise & 15 & $67.23 \pm 7.04^{1}$ \\
High-intensity exhaustive exercise & 5 & $80.32 \pm 8.36^{1,2}$ \\
Pre-exercise & 5 & $76.39 \pm 4.01^{1,2}$ \\
Immediate post-exercise & 5 & \\
24 h post-exercise & 5
\end{tabular}

${ }^{1}$ Compared with controls, $\mathrm{P}<0.05 ;{ }^{2}$ compared with pre-exercise, $\mathrm{P}<0.05$; $\mathrm{NO}=$ nitrous oxide.

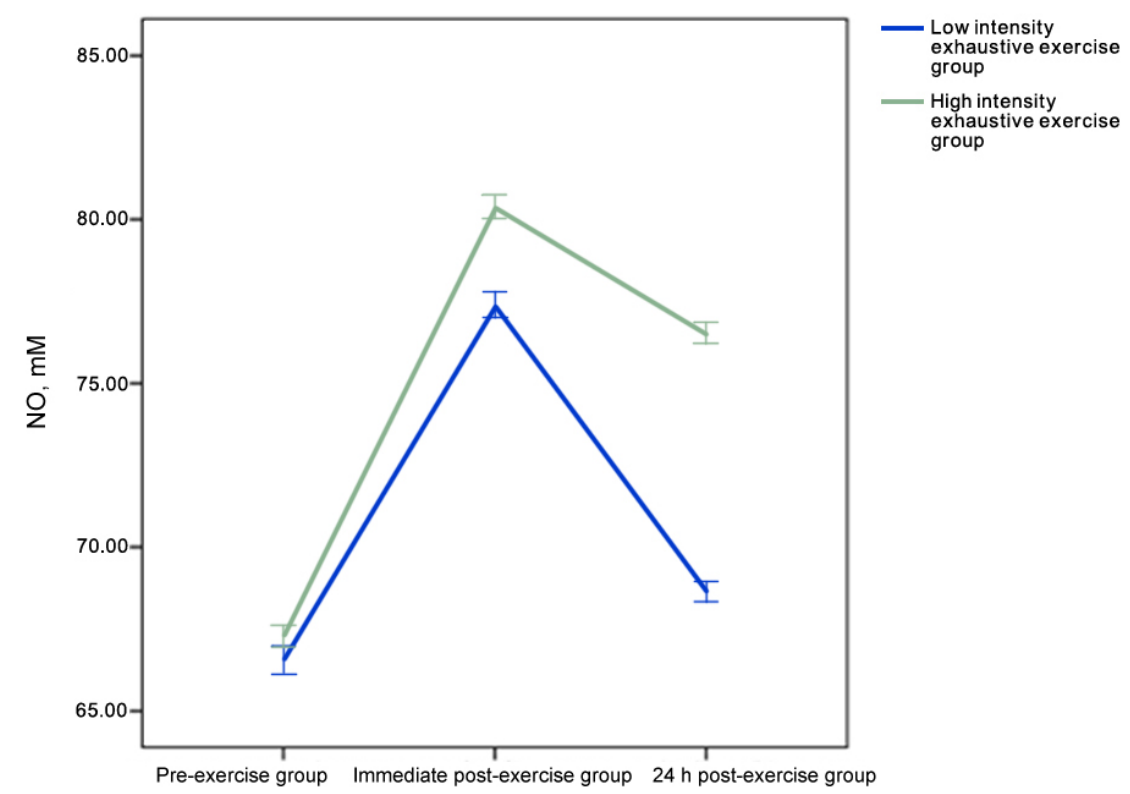

Figure 3. Nitric oxide (NO) levels in the low- and high-intensity exhaustive exercise groups.

\section{DISCUSSION}

A recent study (Bist and Bhatt, 2010) found that the occurrence and development of knee osteoarthritis is the result of the synergy of many factors. Although the pathogenesis of knee osteoarthritis is still not well understood, the role of free radicals in causing damage to the articular cartilage has become generally accepted. Under general pathological conditions of knee osteoarthritis, overwhelming amount of free radicals can inhibit cartilage cell proliferation through the inhibition of DNA synthesis as well as damage the metabolic synthesis of the collagen matrix and proteoglycans, thus accelerating cartilage matrix degradation and eventual cartilage cell death. In turn, loss of cartilage cells reduces the overall strength and elasticity of the cartilage leading to knee osteoarthritis degenerative disease with joint pain and movement limitation (Andriacchi and Favre, 2014). 
SOD is a main free radical scavenger that can significantly reduce free radical damage to the body. SOD is an active substance generated by living organisms which can eliminate the harmful materials such as free radicals produced during normal oxygen metabolism. SOD can react with oxygen free radicals to generate hydrogen peroxide; the latter is converted into water by the organism and excluded. Thus, SOD activity represents the free radical scavenging ability of the body: higher SOD activity represents greater free radical scavenging ability (Marks, 2014). Free radical release is enhanced when the intensity of motion increases; lipid peroxidation and bodily damage are also concomitantly increased. Under normal conditions, SOD release would also gradually increase to maintain the balance between oxidation and antioxidation at this time.

A previous study (Gan et al., 2014) had shown that SOD damage could be identified in the process of knee osteoarthritis pathogenesis and was significantly positively correlated with its severity. Our results revealed that SOD levels were significantly lower in the knee osteoarthritis rat model group than in the control group. Figure 1 shows that SOD levels immediately after the exhaustive exercise in both the low- and high-intensity groups were significantly lower than those in the quiet and pre-exercise groups. SOD decreased immediately after exhaustive exercise in both the low and high-intensity groups, which might be caused by suppression of SOD activity due to the rapid rise of the free radical level. Furthermore, after 24-h recovery, the SOD level in the low-intensity exhaustive exercise group returned to the baseline as defined by the quiet group, whereas it was still significantly lower in the high-intensity exercise group at this time. This finding showed that SOD levels recovered slowly in the high-intensity group which might be related to the larger amounts of damage to the body generated by this regime (Smink et al., 2014).

In the clinic, MDA and SOD have often been treated as matching indicators, of which the SOD level was associated with free radical scavenging ability, whereas MDA reflected the degree of damage caused by free radicals to the cells. During the intensive exercise process, oxygen consumption has been shown to elevate sharply. Enhancement of mitochondrial activity has also been demonstrated, promoting a free radical chain reaction. When the level of free radicals was high, it was found that SOD failed to effectively remove them, leading to lipid peroxidation and cell damage (Yang et al., 2014). Therefore, as a lipid peroxidation product, MDA can not only influence the degree of cell damage, but also reflects the severity of lipid peroxidation.

In the present study, the MDA level was higher in the quiet group than in the controls, which revealed that the MDA level increased in the serum of rats with knee osteoarthritis. The MDA levels in both the low- and high-intensity groups immediately after the exhaustive exercise were significantly higher than that in pre-exercise group, indicating that exhaustion exercise aggravated lipid peroxidation and generated a dramatic increase in MDA level. This finding was consistent with previous research (Killian et al., 2014). Subsequently, the MDA level in the low-intensity exhaustive exercise group decreased with no statistical significant difference at $24 \mathrm{~h}$ post-exercise with the quiet and pre-exercise groups. On the other hand, although the MDA level also declined gradually in the high intensity exercise group, it was still higher than baseline at this time. High-intensity exhaustive exercise might increase cell damage leading to a significant change in cell membrane permeability. Meanwhile, highintensity exhaustive exercise might also decrease the free radical scavenging ability of SOD, thereby slowing the decrease in the MDA level (Wang et al., 2014). 
The occurrence and developmental process of knee osteoarthritis and cartilage cell degeneration would be expected to release a large quantity of active factors and inflammatory mediators into the synovial membrane and the synovial fluid. The balance between active factors and inflammatory mediators has also been shown to play a decisive role in cartilage cell damage (Foster et al., 2014). Among these, suspect cell factors primarily included tumor necrosis factor alpha (TNF- $\alpha$ ), and interleukin 1 alpha (IL- $1 \alpha)$ and 1 beta (IL-1 $\beta$ ), among others. IL- $1 \alpha$ and IL- $1 \beta$ concentrations have been shown to increase under the pathological conditions of osteoarthritis; this increase improved the fibrinolytic enzyme activity, destroyed the cartilage matrix, and increased the incidence of synovial thickening and bone invasion (van Baarsen et al., 2014). In addition, TNF- $\alpha$ has been demonstrated to have an inhibitory effect on matrix repair, so as to accelerate the degeneration of articular cartilage. In turn, NO is an important inflammatory mediator in knee osteoarthritis progression, acting through increased induction of nitric oxide synthase (iNOS) (Ye et al., 2014). In the present study, the NO levels increased in the quiet and the low- and high-intensity exhaustive exercise groups compared with the control group, consistent with findings from clinical studies (Tanaka et al., 2014).

High NO levels have been shown to have a marked inhibitory effect on cartilage cell proliferation. It can induce cartilage cell apoptosis and prevent cartilage matrix synthesis leading to cartilage destruction. At the same time, IL- $1 \beta$ can also accelerate the synthesis and secretion of matrix metalloproteinase 3 in human cartilage cells through the action of NO to aggravate cartilage matrix damage. Therefore, NO also plays an important role in promoting the occurrence and development of knee osteoarthritis (Prior et al., 2014). NO levels immediately after the exhaustive exercise in both the low- and high-intensity groups was dramatically higher than those in the quiet and pre-exercise groups, indicating that NO levels were upregulated after exhaustion exercise. The primary reason for this might be related to the increased muscle blood flow and shear stress caused by vasodilation during exercise, which would likely promote the continuous generation of NO by endothelial cells (Balaganur et al., 2014). After $24 \mathrm{~h}$, the NO level in the low intensity exhaustive exercise group recovered to the quiet and pre-exercise baseline. On the other hand, although the NO level declined in the high-intensity exercise group, it was still higher than that in the quiet and pre-exercise groups at this time. This showed that NO decreased slowly after high intensity exhaustive exercise, which was consistent with the results from clinical research (Laufer et al., 2014). The main reason for this limited decline might be that structural NOS (cNOS) activity was decreased by high-intensity exhaustive exercise, leading to an increased activity of iNOS. In addition, high-intensity exhaustive exercise might have resulted in inflammatory cell infiltration that stimulated neurogliocytes and macrophages. The latter in turn might result in iNOS overexpression in the tissue, leading to the maintenance of a high level of NO $24 \mathrm{~h}$ postexercise (Clement et al., 2014).

In conclusion, the SOD levels decreased, while the MDA and NO levels increased under different intensities of exhaustion exercise in knee osteoarthritis. High-intensity exhaustive exercise might restrain the activity of SOD and cNOS, leading to high levels of NO and lower level of SOD at $24 \mathrm{~h}$ post-exercise, which might exacerbate the knee osteoarthritis pathological state. The future study of different intensities and times of endurance exercise training might allow the identification of movement forms or intensities sufficient to relieve knee osteoarthritis. 


\section{Conflicts of interest}

The authors declare no conflict of interest.

\section{ACKNOWLEDGMENTS}

We would like to thank all the authors. This study did not receive any funding support.

\section{REFERENCES}

Andriacchi TP and Favre J (2014). The nature of in vivo mechanical signals that influence cartilage health and progression to knee osteoarthritis. Curr. Rheumatol. Rep. 16: 463.

Balaganur V, Pathak NN, Lingaraju MC, More AS, et al. (2014). Effect of S-methylisothiourea, an inducible nitric oxide synthase inhibitor, in joint pain and pathology in surgically induced model of osteoarthritis. Connect. Tissue Res: 55: 367-377.

Bedford TG, Tipton CM, Wilson NC, Opplinger RA, et al. (1979). Maximum oxygen consumption of rats and its changes with various experimental procedures. J. Appl. Physiol. Respir. Environ. Exerc. Physiol. 47: 1278-1283.

Bist R and Bhatt DK (2010). Augmentation of cholinesterases and ATPase activities in the cerebellum and pons-medulla oblongata, by a combination of antioxidants (resveratrol, ascorbic acid, alpha-lipoic acid and vitamin E), in acutely lindane intoxicated mice. J. Neurol. Sci. 296: 83-87.

Clément J, Hagemeister N, Dumas R, Khanhonou M, et al. (2014). Influence of biomechanical multi-joint models used in global optimisation to estimate healthy and osteoarthritis knee kinematics. Comput. Methods Biomech. Biomed. Engin. 17: 76-77.

Foster NE, Healey EL, Holden MA, Nicholls, E, et al. (2014). A multicentre, pragmatic, parallel group, randomised controlled trial to compare the clinical and cost-effectiveness of three physiotherapy-led exercise interventions for knee osteoarthritis in older adults: the BEEP trial protocol (ISRCTN: 93634563). BMC Musculoskelet. Disord. 15: 254.

Gan HS, Tan TS, Wong LX, Tham WK, et al. (2014). Interactive knee cartilage extraction using efficient segmentation software: Data from the osteoarthritis initiative. Biomed. Mater. Eng. 24: 3145-3157.

Killian ML, Haut RC and Haut Donahue TL (2014). Acute cell viability and nitric oxide release in lateral menisci following closed-joint knee injury in a lapine model of post-traumatic osteoarthritis. BMC Musculoskelet. Disord. 15: 297.

Laufer Y, Shtraker H and Elboim Gabyzon M (2014). The effects of exercise and neuromuscular electrical stimulation in subjects with knee osteoarthritis: a 3-month follow-up study. Clin. Interv. Aging 9: 1153-1161.

Lu X, Ma L, Ruan L, Kong Y, et al. (2010). Resveratrol differentially modulates inflammatory responses of microglia and astrocytes. J. Neuroinflammation 7: 46.

Panicker S, Borgia J, Fhied C, Mikecz K, et al. (2009). Oral glucosamine modulates the response of the liver and lymphocytes of the mesenteric lymph nodes in a papain-induced model of joint damage and repair. Osteoarthritis Cartilage 17: 1014-1021.

Prior M J, Harrison DD and Frustaci ME (2014). A randomized, double-blind, placebo-controlled 12 week trial of acetaminophen extended release for the treatment of signs and symptoms of osteoarthritis. Curr. Med. Res. Opin. 30: 2377-2387.

Marks R (2014). Perceived health status of women with knee osteoarthritis: a cross-sectional study of the relationships of age, body mass, pain and walking limitations. Open Orthop. J. 8: 255-263.

Smink AJ, Dekker J, Vliet Vlieland TP, Swierstra BA, et al. (2014). Health care use of patients with osteoarthritis of the hip or knee after implementation of a stepped-care strategy: an observational study. Arthritis Care Res. 66: 817-827.

Tanaka R, Ozawa J, Kito N and Moriyama H (2014). Effect of the frequency and duration of land-based therapeutic exercise on pain relief for people with knee osteoarthritis: a systematic review and meta-analysis of randomized controlled trials. J. Phys. Ther. Sci. 26: 969-975.

Toklu HZ, Sehirli O, Ersahin M, Süleymanoğlu S, et al. (2010). Resveratrol improves cardiovascular function and reduces oxidative organ damage in the renal, cardiovascular and cerebral tissues of two-kidney, one-clip hypertensive rats. $J$. Pharm. Pharmacol. 62: 1784-1793.

van Baarsen L, Lebre MC, van der Coelen D, Aarrass S, et al. (2014). Heterogeneous expression pattern of interleukin17A (IL-17A), IL-17F and their receptors in synovium of rheumatoid arthritis, psoriatic arthritis and osteoarthritis: possible explanation for nonresponse to anti-IL-17 therapy? Arthritis Res. Ther. 16: 426. 
Wang C, Zhong D, Liao Q, Kong L, et al. (2014). Procalcitonin levels in fresh serum and fresh synovial fluid for the differential diagnosis of knee septic arthritis from rheumatoid arthritis, osteoarthritis and gouty arthritis. Exp. Ther. Med. 8: 1075-1080.

Yang X, Zhao J, He Y and Huangfu X (2014). Screening for characteristic genes in osteoarthritis induced by destabilization of the medial meniscus utilizing bioinformatics approach. J. Musculoskelet. Neuronal Interact. 14: 343-348.

Ye J, Cai S, Zhong W, Cai S, et al. (2014). Effects of tai chi for patients with knee osteoarthritis: a systematic review. $J$. Phys. Ther. Sci. 26: 1133-1137. 